\title{
Discordant SARS-CoV-2 PCR and Rapid Antigen Test Results When Infectious: A December 2021 Occupational Case Series
}

\author{
Blythe Adamson, PhD, MPH ${ }^{1,2,3}$, Robby Sikka, MD ${ }^{1}$, Anne L. Wyllie, $\mathrm{PhD}^{1,4}$, \\ Prem Premsrirut, MD, $\mathrm{PhD}^{1,5,6}$
}

${ }^{1}$ COVID-19 Sports and Society Working Group, USA; ${ }^{2}$ Infectious Economics, LLC, New York, NY, USA $;^{3}$ The Comparative Health Outcomes, Policy and Economics Institute, School of Pharmacy, University of Washington, Seattle, WA, USA; ${ }^{4}$ Yale School of Public Health, New Haven, CT, USA; 5 Mirimus Laboratories, Brooklyn, NY, USA; ${ }^{6}$ State University of New York Downstate Medical Center, Brooklyn, NY, USA

\author{
Corresponding Author: \\ Blythe Adamson, $\mathrm{PhD}, \mathrm{MPH}$ \\ Email: blythe@infectiouseconomics.com
}

\begin{abstract}
The performance of Covid-19 diagnostic tests must continue to be reassessed with new variants of concern. The objective of this study was to describe the discordance in saliva SARS-CoV-2 PCR and nasal rapid antigen test results during the early infectious period. We identified a high-risk occupational case cohort of 30 individuals with daily testing during an Omicron outbreak in December 2021. Based on viral load and transmissions confirmed through epidemiological investigation, most Omicron cases were infectious for several days before being detectable by rapid antigen tests.
\end{abstract}

Key words: SARS-CoV-2, Covid-19, Surveillance, Testing 
medRxiv preprint doi: https://doi.org/10.1101/2022.01.04.22268770; this version posted January 5, 2022. The copyright holder for this preprint (which was not certified by peer review) is the author/funder, who has granted medRxiv a license to display the preprint in perpetuity.

It is made available under a CC-BY 4.0 International license.

Routine workplace Covid-19 surveillance testing has been key to reopening in-person businesses with job functions that have high-risk for SARS-CoV-2 transmission. Yet, the real world performance of Covid-19 diagnostic tests needs to be reassessed for each new variant of concern. The FDA recently updated guidance stating that antigen tests may be less sensitive for the detection of Omicron than previous SARS-CoV-2 variants. ${ }^{1}$ However, laboratory experiments cannot fully replace clinical study evaluations using patient samples through the course of SARS-CoV-2 infection. Omicron has been shown to infect faster and more efficiently than Delta in human bronchus, but with less severe infection in lung, ${ }^{2}$ translating to symptom increase of sore throats and decrease of loss of taste and smell, better detected by saliva than nasal swabs. ${ }^{3-5}$ To date, the viral dynamics and test performance in the Omicron early infection period have not been described in detail, as it requires cohorts receiving near daily testing to identify cases prior to symptom onset.

\section{METHODS}

This retrospective cohort study identified individuals in occupational safety programs who were diagnosed with Covid-19 between December 1-31, 2021 during Omicron outbreaks at five workplaces in New York, NY, Los Angeles, CA, and San Francisco, CA. The populations were fully vaccinated by employer mandate and highly boosted by choice. To isolate the window of acute infection, cases were included if they were receiving daily testing at the time of diagnosis, had paired SARS-CoV-2 quantitative reverse-transcriptase polymerase chain reaction (RT-qPCR) test results and rapid antigen test results on Day 0 or 1 relative to first positive specimen collection date and were excluded if missing a recent negative test. The primary outcome of interest was discordance between saliva PCR and nasal rapid antigen test results during the early period with viral load levels corresponding to infectious risk of transmission. A Kaplan Meier analysis was performed to estimate the median time from first PCR positive to first rapid antigen positive test result. 
medRxiv preprint doi: https://doi.org/10.1101/2022.01.04.22268770; this version posted January 5, 2022. The copyright holder for this preprint (which was not certified by peer review) is the author/funder, who has granted medRxiv a license to display the preprint in perpetuity.

It is made available under a CC-BY 4.0 International license.

\section{RESULTS}

Among hundreds of workplace-detected Covid-19 cases in December 2021, we identified 30 individuals with 62 matched pairs of rapid antigen and positive PCR results from specimens collected at the same time. The S-gene dropout associated with Omicron was observed in 29 of 30 cases. Viral dynamics and discordance in test results are shown in Figure 1. Four cases were confirmed to have transmitted the virus between false-negative antigen tests, with saliva PCR cycle threshold $(\mathrm{Ct})$ values between 23-28 for the $\mathrm{N}$ gene. On Days 0 and 1, all rapid antigen tests produced false-negative results, despite 28 of 30 pairs having infectious viral load within the range of confirmed Omicron transmissions in the cohort $(\mathrm{Ct}<29)$. The median time from first positive PCR to first detectable antigen positive was 3 days $(95 \%$ Confidence Interval: 2-NA). After infection was detected, a subgroup $(\mathrm{n}=5)$ who received daily saliva PCR, nasal swab PCR, and nasal swab rapid antigen testing showed viral load peaked in saliva 1-2 days before nasal tests (Supplemental Table 1). All individuals in the cohort developed symptoms within two days of the first PCR positive test.

\section{DISCUSSION}

We found that rapid antigen tests lagged in the ability to detect Covid-19 during an early period of disease when most individuals were infectious with Omicron and four transmissions were confirmed. The policy implication is that rapid antigen tests may not be as fit-for-purpose in routine workplace screening to prevent asymptomatic spread of Omicron, compared to prior variants, ${ }^{6}$ given the shorter time from exposure to infectiousness and lower infectious doses sufficient for transmission. These findings are consistent with population-level Omicron epidemiology studies showing shorter serial intervals between cases and faster rates of community spread. Despite the small numbers of individuals included in this study, the findings are uniquely valuable because of the early detection of Omicron infection in frequent workplace Covid-19 testing to prevent spread. In real-world antigen testing, the limit of detection was substantially lower than manufacturers have reported to the FDA based on laboratory validation. 


\section{Sources of Funding}

No funding was received to support this study.

\section{Potential Conflicts}

BJA reports receiving consultancy fees for safety advising several workplaces included in this study; serving as an unpaid board member, SalivaDirect; being a paid employee of Flatiron Health, outside the submitted work. RS reports serving as an unpaid board member, SalivaDirect, outside of this work. ALW reports serving as an unpaid board member of SalivaDirect, outside of this work. PP reports being a paid employee of Mirimus Laboratory.

\section{Acknowledgements}

We thank the COVID-19 Sports and Society Working Group, workplace COVID Safety Managers, Harlan Krumholz, MD and Nathan Grubaugh, PhD from Yale University, and the operational team, including Charlene Speyer and Lisa Kussell from Infectious Economics. 
medRxiv preprint doi: https://doi.org/10.1101/2022.01.04.22268770; this version posted January 5, 2022. The copyright holder for this preprint (which was not certified by peer review) is the author/funder, who has granted medRxiv a license to display the preprint in perpetuity.

It is made available under a CC-BY 4.0 International license .

\section{References}

1. US Food and Drug Administration. FDA Omicron Variant Antigen test performance waning.

"Omicron Variant: Impact on Antigen Diagnostic Tests (As of 12/28/2021)"

https://www.fda.gov/medical-devices/coronavirus-covid-19-and-medical-devices/sars-cov-2-viral -mutations-impact-covid-19-tests\#omicronvariantimpact. Accessed December 31, 2021.

2. LKS Faculty of Medicine of The University of Hong Kong. HKUMed finds Omicron

SARS-CoV-2 can infect faster and better than delta in human bronchus but with less severe infection in lung. 15 December 2021.

www.med.hku.hk/en/news/press/20211215-omicron-sars-cov-2-infection. Accessed December $31,2021$.

3. Teo AKJ, Choudhury Y, Tan IB, Cher CY, Chew SH, Wan ZY, Cheng LTE, Oon LLE, Tan MH, Chan KS, Hsu LY. Saliva is more sensitive than nasopharyngeal or nasal swabs for diagnosis of asymptomatic and mild COVID-19 infection. Sci Rep. 2021 Feb 4;11(1):3134. doi:

10.1038/s41598-021-82787-z. Erratum in: Sci Rep. 2021 Jun 9;11(1):12538. PMID: 33542443;

PMCID: PMC7862309.

4. Wyllie AL, Fournier J, Casanovas-Massana A, Campbell M, Tokuyama M, Vijayakumar P, Warren JL, Geng B, Muenker MC, Moore AJ, Vogels CBF, Petrone ME, Ott IM, Lu P, Venkataraman A, Lu-Culligan A, Klein J, Earnest R, Simonov M, Datta R, Handoko R, Naushad N, Sewanan LR, Valdez J, White EB, Lapidus S, Kalinich CC, Jiang X, Kim DJ, Kudo E, Linehan M, Mao T, Moriyama M, Oh JE, Park A, Silva J, Song E, Takahashi T, Taura M, Weizman OE, Wong P, Yang Y, Bermejo S, Odio CD, Omer SB, Dela Cruz CS, Farhadian S, Martinello RA, Iwasaki A, Grubaugh ND, Ko AI. Saliva or Nasopharyngeal Swab Specimens for Detection of SARS-CoV-2. N Engl J Med. 2020 Sep 24;383(13):1283-1286. doi:

10.1056/NEJMc2016359. Epub 2020 Aug 28. PMID: 32857487 ; PMCID: PMC7484747. 
5. Marais G, Hsiao NY, Iranzadeh A, Doolabh D, Enoch A, Chu CY, Williamson C, Brink A, Hardie D. Saliva swabs are the preferred sample for Omicron detection. medRxiv 2021.12.22.21268246; doi: https://doi.org/10.1101/2021.12.22.21268246. Accessed 4 Jan 2021.

6. Kissler SM, Fauver JR, Mack C, Olesen SW, Tai C, Shiue KY, Kalinich CC, Jednak S, Ott IM, Vogels CBF, Wohlgemuth J, Weisberger J, DiFiori J, Anderson DJ, Mancell J, Ho DD, Grubaugh ND, Grad YH. Viral dynamics of acute SARS-CoV-2 infection and applications to diagnostic and public health strategies. PLoS Biol. 2021 Jul 12;19(7):e3001333. doi:

10.1371/journal.pbio.3001333. PMID: 34252080; PMCID: PMC8297933. 
medRxiv preprint doi: https://doi.org/10.1101/2022.01.04.22268770; this version posted January 5, 2022. The copyright holder for this preprint (which was not certified by peer review) is the author/funder, who has granted medRxiv a license to display the preprint in perpetuity.

It is made available under a CC-BY 4.0 International license.

Figure 1. Discordance in PCR and Rapid Antigen Test Results

Shown are Cycle Threshold $(\mathrm{Ct})$ counts and rapid antigen test results from paired samples of infected patients (Panel A). Also shown is the Kaplan Meier analysis of time from positive PCR to positive rapid antigen test (Panel B).

A)

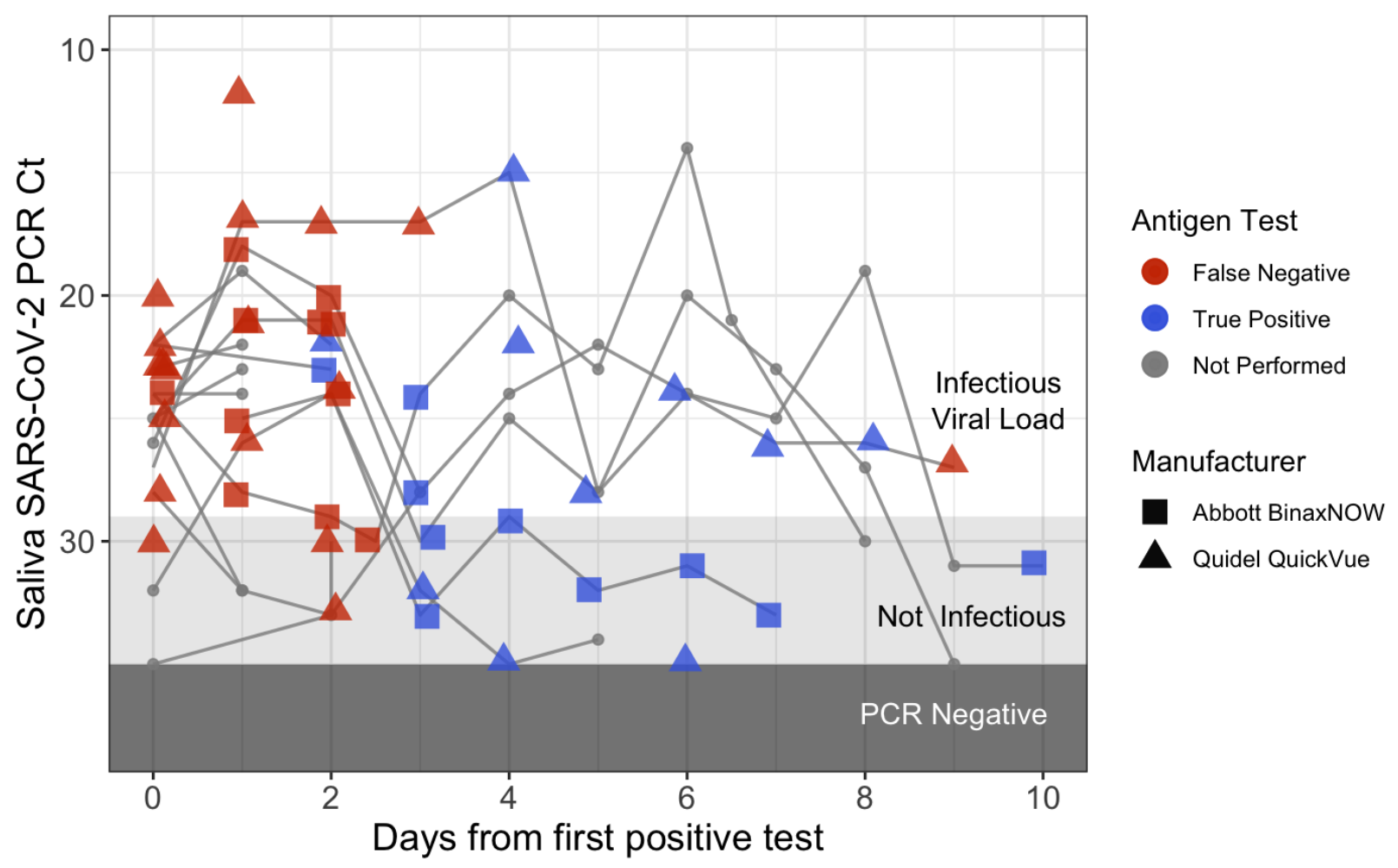

B)

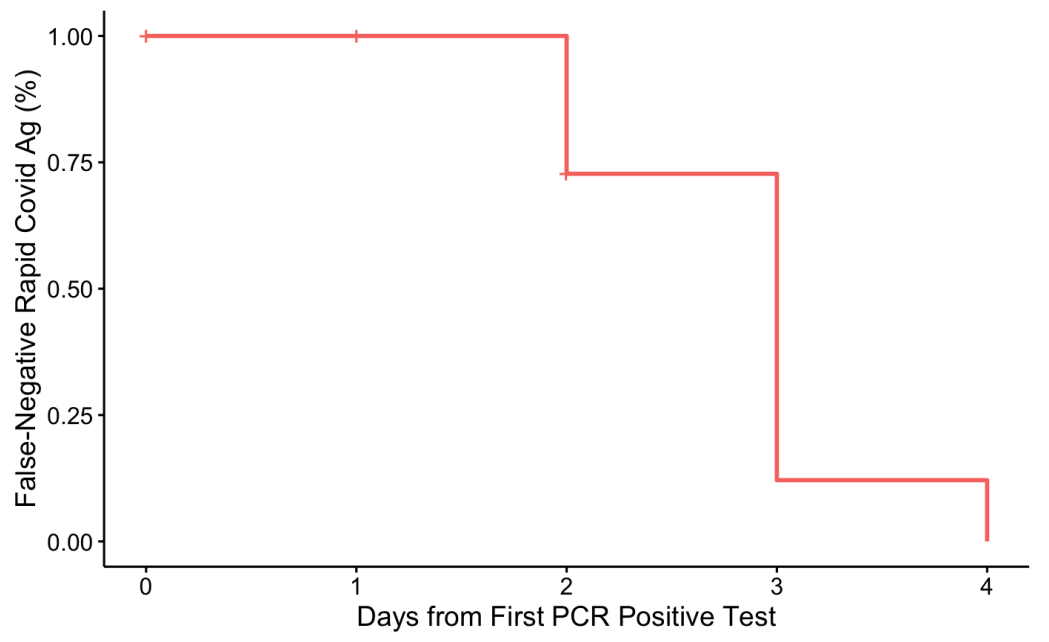




\section{Supplementary Appendix}

\section{Study Design}

The data reported here represent a convenience sample of employees at a selection of workplaces that partner with Infectious Economics LLC for Covid-19 prevention, including corporate offices, entertainment, retail trade, and manufacturing. The study period ran December 1, 2021 to December 31, 2021. Clinical samples were obtained by self-collection observed by a trained COVID Safety Manager. Employer-provided testing was preventatively provided to enable in-person workplace safety during times of high community prevalence and/or recent COVID exposures in the workplace. Because the sensitivity of tests may vary during the course of an infection, we evaluated concordance of PCR and rapid antigen test results in matched samples over time.

\section{Study Oversight}

In accordance with the guidelines, this work with de-identified samples was approved for research not involving human subjects by the SUNY Downstate Institutional Review Board \& Privacy Board (1603504-6) under the title "Pilot program for instituting massive COVID19 surveillance screening in schools and the workplace."

\section{Data Availability and Code}

All de-identified data and the code for analysis is available on GitHub at

https://github.com/blythejane/covid safety.

\section{Covid-19 Testing and Sequencing}

The rapid antigen tests kits used were Quidel QuickVue At-Home OTC COVID-19 Test and Abbott BinaxNOW COVID-19 Antigen Self-Test. PCR to detect SARS-CoV-2 RNA was the ThermoFisher 
medRxiv preprint doi: https://doi.org/10.1101/2022.01.04.22268770; this version posted January 5, 2022. The copyright holder for this preprint (which was not certified by peer review) is the author/funder, who has granted medRxiv a license to display the preprint in perpetuity.

It is made available under a CC-BY 4.0 International license .

Combo Kit, allowing for the ability to detect an S-gene dropout. The time from specimen collection to results returned was $<30$ minutes for antigen tests and ranged 6-12 hours for PCR. A subset of specimens received whole genome sequencing in a broader epidemiology investigation of clusters to further improve workplace safety policies. Samples were classified as Omicron or Delta based on whole genome sequencing data, diagnostic PCR target failures, and sampling dates. In whole genome sequencing, RNA was extracted and confirmed as SARS-CoV- 2 positive by RT-qPCR with the Thermo Fisher TaqPath SARS-CoV-2 assay. Next Generation Sequencing with the Illumina COVIDSeq ARTIC primer set2 was used for viral amplification.

\section{Outcomes}

The primary outcome of interest was concordance between PCR and rapid antigen test results, dependent on time and $\mathrm{Ct}$ values corresponding to infectiousness. We defined the index as the specimen collection date for the first PCR test with detectable SARS-CoV-2 with $\mathrm{Ct}<35$, the event being the first positive rapid antigen test result, and censoring at the most recent antigen test date. Based on observed transmission events in these workplaces that were confirmed by contact tracing and genomic epidemiology investigation, we defined infectious viral load as corresponding to $\mathrm{Ct}$ values $<29$ in this analysis.

\section{Statistical analysis}

A Kaplan Meier analysis was performed to estimate the median time from PCR positive to rapid antigen positive and time-dependent probability of a false negative rapid antigen test. We defined the index as the specimen collection date for the first PCR test with detectable SARS-CoV-2 with $\mathrm{Ct}<35$, the event being the first positive rapid antigen test result, and censoring at the most recent antigen test date. Analyses were conducted in R 4.1.2. 
Supplementary Table 1. Covid-19 cases with discordant test results from paired PCR and antigen tests in workplace safety programs

\begin{tabular}{|c|c|c|c|c|c|c|c|c|c|c|c|}
\hline & & & NDICATOR & & & AB PC & & & ANTIGEN TEST & & RAPID MOLECULAR \\
\hline Case & Day & $\begin{array}{l}\text { PCR \& } \\
\text { Antigen } \\
\text { Aligned }\end{array}$ & $\begin{array}{l}\text { Infectious } \\
(\mathrm{Ct}<29)\end{array}$ & $\begin{array}{c}\text { High Risk } \\
\text { Flag*** }\end{array}$ & $\begin{array}{c}\mathbf{C t} \\
\text { Saliva }\end{array}$ & $\begin{array}{c}\mathbf{C t} \\
\text { Nasal }\end{array}$ & PCR Result & $\begin{array}{c}\text { Rapid } \\
\text { Antigen } \\
\text { Result }\end{array}$ & $\begin{array}{c}\text { Manufacturer of } \\
\text { Antigen Test }\end{array}$ & $\begin{array}{c}\text { Rapid } \\
\text { Molecular } \\
\text { Result }\end{array}$ & $\begin{array}{c}\text { Manufacturer of Rapid } \\
\text { Molecular Test }\end{array}$ \\
\hline A & 0 & & & & & & Positive & & & & \\
\hline A & 1 & FALSE & TRUE & TRUE & 12 & & Positive & Negative & Quidel QuickVue & & \\
\hline $\mathrm{B}$ & -2 & & FALSE & & & & Negative & & & & \\
\hline B & -1 & & & & & & & & & Negative & Lucira Check-It \\
\hline $\mathrm{B}$ & 0 & FALSE & TRUE & TRUE & 27 & & Positive & Negative & Abbott BinaxNOW & Positive & Cue COVID-19 \\
\hline $\mathrm{B}$ & 1 & FALSE & TRUE & TRUE & 17 & & Positive & Negative & Quidel QuickVue & & \\
\hline $\mathrm{B}$ & 2 & FALSE & TRUE & TRUE & 17 & & Positive & Negative & Quidel QuickVue & & \\
\hline $\mathrm{B}$ & 3 & FALSE & TRUE & TRUE & 17 & & Positive & Negative & Quidel QuickVue & & \\
\hline $\mathrm{B}$ & 4 & TRUE & TRUE & FALSE & 15 & & Positive & Positive & Quidel QuickVue & & \\
\hline B & 5 & TRUE & TRUE & FALSE & 28 & & Positive & Positive & Quidel QuickVue & & \\
\hline B & 6 & TRUE & TRUE & FALSE & 24 & & Positive & Positive & Quidel QuickVue & & \\
\hline $\mathrm{B}$ & 7 & TRUE & TRUE & FALSE & 26 & & Positive & Positive & Quidel QuickVue & & \\
\hline B & 8 & TRUE & TRUE & FALSE & 26 & & Positive & Positive & Quidel QuickVue & & \\
\hline B & 9 & FALSE & TRUE & TRUE & 27 & & Positive & Negative & Quidel QuickVue & & \\
\hline $\mathrm{C}$ & 0 & & TRUE & & 26 & & Positive & & & & \\
\hline $\mathrm{C}$ & 1 & FALSE & TRUE & TRUE & 18 & 32 & Positive & Negative & Abbott BinaxNOW & & \\
\hline $\mathrm{C}$ & 2 & FALSE & TRUE & TRUE & 20 & 31 & Positive & Negative & Abbott BinaxNOW & & \\
\hline $\mathrm{C}$ & 3 & TRUE & TRUE & FALSE & 28 & 27 & Positive & Positive & Abbott BinaxNOW & & \\
\hline $\mathrm{C}$ & 4 & TRUE & TRUE & & 24 & 23 & Positive & & & & \\
\hline $\mathrm{C}$ & 5 & TRUE & TRUE & & 22 & 18 & Positive & & & & \\
\hline $\mathrm{C}$ & 6 & TRUE & TRUE & & 24 & 16 & Positive & & & & \\
\hline $\mathrm{C}$ & 7 & TRUE & TRUE & & 25 & 24 & Positive & & & & \\
\hline $\mathrm{C}$ & 8 & TRUE & TRUE & & 19 & 20 & Positive & & & & \\
\hline
\end{tabular}




\begin{tabular}{|c|c|c|c|c|c|c|c|c|c|c|c|}
\hline $\mathrm{C}$ & 9 & TRUE & TRUE & & 31 & 28 & Positive & & & & \\
\hline $\mathrm{C}$ & 10 & TRUE & FALSE & FALSE & 31 & 27 & Positive & Positive & Abbott BinaxNOW & & \\
\hline $\mathrm{D}$ & -1 & & & & & & & Negative & Quidel QuickVue & & \\
\hline $\mathrm{D}$ & 0 & FALSE & TRUE & TRUE & 20 & & Positive & Negative & Quidel QuickVue & & \\
\hline $\mathrm{E}^{*}$ & -1 & & & & & & & Negative & Quidel QuickVue & & \\
\hline $\mathrm{E}^{*}$ & 0 & FALSE & TRUE & TRUE & 20 & & Positive & Negative & Quidel QuickVue & & \\
\hline $\mathrm{F}$ & -1 & & FALSE & & & & & & & Negative & Lucira Check-It \\
\hline $\mathrm{F}$ & 0 & & & & & & & Negative & Quidel QuickVue & Positive & Lucira Check-It \\
\hline $\mathrm{F}$ & 1 & FALSE & TRUE & TRUE & 21 & & Positive & Negative & Quidel QuickVue & & \\
\hline $\mathrm{F}$ & 2 & & TRUE & & & & & Positive & Quidel QuickVue & & \\
\hline $\mathrm{F}$ & 4 & TRUE & TRUE & FALSE & 22 & & Positive & Positive & Quidel QuickVue & & \\
\hline $\mathrm{F}$ & 5 & FALSE & FALSE & FALSE & & & Negative & Positive & Quidel QuickVue & & \\
\hline $\mathrm{F}$ & 6 & TRUE & FALSE & FALSE & 35 & & Positive & Positive & Quidel QuickVue & & \\
\hline $\mathrm{F}$ & 8 & & & & & & & Negative & Quidel QuickVue & Positive & Lucira Check-It \\
\hline G & -1 & & FALSE & & & & & & & Negative & Lucira Check-It \\
\hline $\mathrm{G}$ & 0 & & & & & & & & & Positive & Lucira Check-It \\
\hline $\mathrm{G}$ & 2 & FALSE & TRUE & TRUE & 21 & & Positive & Negative & Abbott BinaxNOW & Positive & Cue COVID-19 \\
\hline $\mathrm{H}^{*}$ & 0 & & TRUE & & 25 & & Positive & & & & \\
\hline $\mathrm{H}^{*}$ & 1 & FALSE & TRUE & TRUE & 21 & 34 & Positive & Negative & Abbott BinaxNOW & & \\
\hline $\mathrm{H}^{*}$ & 2 & FALSE & TRUE & TRUE & 21 & 29 & Positive & Negative & Abbott BinaxNOW & & \\
\hline $\mathrm{H}^{*}$ & 3 & TRUE & FALSE & FALSE & 30 & 23 & Positive & Positive & Abbott BinaxNOW & & \\
\hline $\mathrm{H}^{*}$ & 4 & & TRUE & & 25 & 13 & Positive & & & & \\
\hline $\mathrm{H}^{*}$ & 5 & & TRUE & & 28 & 15 & Positive & & & & \\
\hline $\mathrm{H}^{*}$ & 6 & & TRUE & & 20 & 20 & Positive & & & & \\
\hline $\mathrm{H}^{*}$ & 7 & & TRUE & & 23 & 25 & Positive & & & & \\
\hline $\mathrm{H}^{*}$ & 8 & & TRUE & & 27 & 29 & Positive & & & & \\
\hline $\mathrm{H}^{*}$ & 9 & & FALSE & & 35 & 31 & Negative & & & & \\
\hline
\end{tabular}




\begin{tabular}{|c|c|c|c|c|c|c|c|c|c|c|c|}
\hline $\mathrm{H}^{*}$ & 10 & TRUE & FALSE & FALSE & & 34 & Negative & Negative & Abbott BinaxNOW & & \\
\hline I & 0 & FALSE & TRUE & TRUE & 21 & & Positive & Negative & Quidel QuickVue & & \\
\hline $\mathrm{J}$ & -3 & TRUE & FALSE & FALSE & & & Negative & Negative & Quidel QuickVue & & \\
\hline $\mathrm{J}$ & 0 & FALSE & TRUE & TRUE & 22 & & Positive & Negative & Quidel QuickVue & Positive & Abbott ID NOW \\
\hline $\mathrm{J}$ & 1 & & TRUE & & 19 & & Positive & & & & \\
\hline $\mathrm{J}$ & 2 & TRUE & TRUE & FALSE & 22 & & Positive & Positive & Quidel QuickVue & & \\
\hline $\mathrm{K}$ & -1 & & & & & & & Negative & Quidel QuickVue & & \\
\hline $\mathrm{K}$ & 0 & FALSE & TRUE & TRUE & 22 & & Positive & Negative & Quidel QuickVue & & \\
\hline K & 2 & TRUE & TRUE & FALSE & 23 & & Positive & Positive & Abbott BinaxNOW & Positive & Lucira Check-It \\
\hline $\mathrm{L}$ & -3 & & FALSE & & & & Negative & & & & \\
\hline $\mathrm{L}$ & 0 & FALSE & TRUE & TRUE & 23 & & Positive & Negative & Quidel QuickVue & & \\
\hline $\mathrm{L}$ & 4 & & FALSE & & & & Negative & & & & \\
\hline $\mathrm{M}$ & 0 & FALSE & TRUE & TRUE & 23 & & Positive & Negative & Quidel QuickVue & & \\
\hline $\mathrm{N}^{*}$ & -1 & & & & & & & Negative & Quidel QuickVue & & \\
\hline $\mathrm{N}^{*}$ & 0 & FALSE & TRUE & TRUE & 23 & & Positive & Negative & Quidel QuickVue & & \\
\hline $\mathrm{N}^{*}$ & 1 & & TRUE & & 22 & & Positive & & & Positive & Lucira Check-It \\
\hline $\mathrm{O}$ & 0 & FALSE & TRUE & TRUE & 23 & & Positive & Negative & Quidel QuickVue & & \\
\hline $\mathrm{P}$ & 0 & FALSE & TRUE & TRUE & 24 & & Positive & Negative & Abbott BinaxNOW & & \\
\hline $\mathrm{P}$ & 1 & FALSE & TRUE & TRUE & 28 & 29 & Positive & Negative & Abbott BinaxNOW & & \\
\hline $\mathrm{P}$ & 2 & FALSE & FALSE & TRUE & 29 & 28 & Positive & Negative & Abbott BinaxNOW & & \\
\hline $\mathrm{P}$ & 2.5 & FALSE & FALSE & TRUE & 30 & 25 & Positive & Negative & Abbott BinaxNOW & & \\
\hline $\mathrm{P}$ & 3 & TRUE & TRUE & FALSE & 24 & 22 & Positive & Positive & Abbott BinaxNOW & & \\
\hline $\mathrm{P}$ & 4 & & TRUE & & 20 & 26 & Positive & & & & \\
\hline $\mathrm{P}$ & 5 & & TRUE & & 23 & 15 & Positive & & & & \\
\hline $\mathrm{P}$ & 6 & & TRUE & & 14 & 15 & Positive & & & & \\
\hline $\mathrm{P}$ & 6.5 & & TRUE & & 21 & 18 & Positive & & & & \\
\hline
\end{tabular}




\begin{tabular}{|c|c|c|c|c|c|c|c|c|c|c|c|}
\hline $\mathrm{P}$ & 8 & & FALSE & & 30 & & Positive & & & & \\
\hline Q & -1 & & FALSE & & & & Negative & & & & \\
\hline Q & 0 & & FALSE & & 32 & & Positive & & & & \\
\hline Q & 1 & FALSE & TRUE & TRUE & 26 & 36 & Positive & Negative & Quidel QuickVue & & \\
\hline Q & 2 & FALSE & TRUE & TRUE & 24 & 35 & Positive & Negative & Quidel QuickVue & & \\
\hline Q & 3 & TRUE & FALSE & FALSE & 32 & 20 & Positive & Positive & Quidel QuickVue & & \\
\hline Q & 4 & TRUE & FALSE & FALSE & 35 & 24 & Positive & Positive & Quidel QuickVue & & \\
\hline $\mathrm{Q}$ & 5 & & FALSE & & 34 & 25 & Positive & & & & \\
\hline $\mathrm{R}$ & -1 & & & & & & & Negative & Quidel QuickVue & & \\
\hline $\mathrm{R}$ & 0 & FALSE & TRUE & TRUE & 24 & & Positive & Negative & Quidel QuickVue & & \\
\hline $\mathrm{R}$ & 1 & & TRUE & & 24 & & Positive & & & Positive & Lucira Check-It \\
\hline $\mathrm{S}$ & 0 & & & & & & & Negative & Abbott BinaxNOW & & \\
\hline $\mathrm{S}$ & 1 & FALSE & TRUE & TRUE & 25 & & Positive & Negative & Abbott BinaxNOW & & \\
\hline S & 2 & FALSE & TRUE & TRUE & 24 & 25 & Positive & Negative & Abbott BinaxNOW & & \\
\hline S & 3 & TRUE & FALSE & FALSE & 33 & 19 & Positive & Positive & Abbott BinaxNOW & & \\
\hline $\mathrm{S}$ & 4 & TRUE & FALSE & FALSE & 29 & 25 & Positive & Positive & Abbott BinaxNOW & & \\
\hline $\mathrm{S}$ & 5 & TRUE & FALSE & FALSE & 32 & 20 & Positive & Positive & Abbott BinaxNOW & & \\
\hline $\mathrm{S}$ & 6 & TRUE & FALSE & FALSE & 31 & 20 & Positive & Positive & Abbott BinaxNOW & & \\
\hline $\mathrm{S}$ & 7 & TRUE & FALSE & FALSE & 33 & 16 & Positive & Positive & Abbott BinaxNOW & & \\
\hline $\mathrm{T}$ & -2 & & & & & & Negative & & & & \\
\hline $\mathrm{T}$ & 0 & FALSE & TRUE & TRUE & 25 & & Positive & Negative & Quidel QuickVue & & \\
\hline $\mathrm{T}$ & 1 & & FALSE & & 32 & & Positive & & & & \\
\hline $\mathrm{T}$ & 2 & & FALSE & & 33 & & Positive & & & & \\
\hline $\mathrm{T}$ & 3 & & TRUE & & 28 & & Positive & & & & \\
\hline $\mathrm{U}$ & -1 & & & & & & & Negative & Quidel QuickVue & & \\
\hline $\mathrm{U}$ & 0 & FALSE & TRUE & TRUE & 25 & & Positive & Negative & Quidel QuickVue & & \\
\hline
\end{tabular}




\begin{tabular}{|c|c|c|c|c|c|c|c|c|c|c|}
\hline $\mathrm{U}$ & 1 & & TRUE & & 23 & Positive & & & Positive & Lucira Check-It \\
\hline V & 0 & FALSE & TRUE & TRUE & 25 & Positive & Negative & Quidel QuickVue & & \\
\hline $\mathrm{W}$ & 0 & FALSE & TRUE & TRUE & 25 & Positive & Negative & Abbott BinaxNOW & Positive & BioReference Laboratory \\
\hline $\mathrm{X}^{*}$ & 0 & FALSE & TRUE & TRUE & 28 & Positive & Negative & Quidel QuickVue & & \\
\hline $\mathrm{Y}$ & 0 & FALSE & TRUE & TRUE & 28 & Positive & Negative & Quidel QuickVue & & \\
\hline $\mathrm{Y}$ & 1 & & FALSE & & 32 & Positive & & & & \\
\hline $\mathrm{Z}$ & 0 & FALSE & TRUE & TRUE & 28 & Positive & Negative & Quidel QuickVue & & \\
\hline AA & 0 & FALSE & FALSE & FALSE & 30 & Positive & Negative & Quidel QuickVue & & \\
\hline $\mathrm{BB}$ & 0 & & FALSE & & 35 & Positive & & & & \\
\hline $\mathrm{BB}$ & 2 & FALSE & FALSE & FALSE & 33 & Positive & Negative & Quidel QuickVue & & \\
\hline $\mathrm{BB}$ & 2 & FALSE & FALSE & FALSE & 30 & Positive & Negative & Quidel QuickVue & & \\
\hline $\mathrm{CC}$ & -1 & & TRUE & & & & Negative & & & \\
\hline $\mathrm{CC}$ & 0 & FALSE & FALSE & FALSE & 34 & Positive & Negative & & & \\
\hline $\mathrm{DD}$ & -2 & & FALSE & & & Negative & & & & \\
\hline $\mathrm{DD}$ & -1 & & FALSE & & & & & & Negative & Lucira Check-It \\
\hline $\mathrm{DD}$ & 0 & FALSE & TRUE & TRUE & & Positive & Negative & Abbott BinaxNOW & Positive & Cue COVID-19 \\
\hline
\end{tabular}

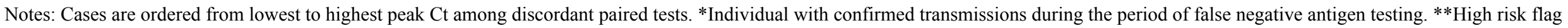

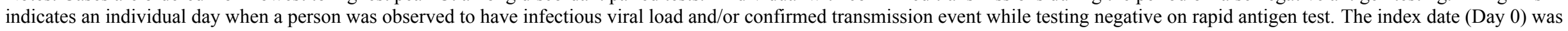

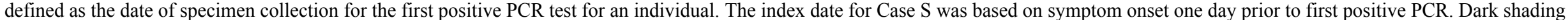

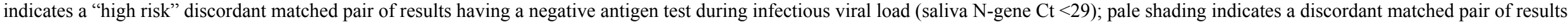

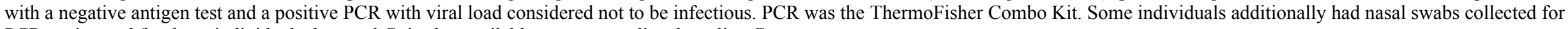
PCR testing and for those individuals the nasal $\mathrm{Ct}$ is also available to contextualize the saliva $\mathrm{Ct}$. 